\title{
APPLYING SUPPORT VECTOR MACHINE TO ELECTRONIC HEALTH RECORDS FOR CANCER CLASSIFICATION
}

\author{
Xudong Zhang \\ Jiehao Xiao \\ Department of Computer Science \\ CUNY Graduate Center \\ 3655 th Ave \\ New York, NY 10016 \\ xzhang5@gradcenter.cuny.edu
}

\author{
Feng Gu \\ Department of Computer Science \\ The College of Staten Island \\ 2800 Victory Boulevard \\ Staten Island, NY 10314 \\ Feng.Gu@csi.cuny.edu
}

\begin{abstract}
An electronic health record (EHR) is a digital record of patients' clinic information and medical records. The EHRs have been receiving more attentions, due to the extensive amount of valuable medical data, which is able to help medical providers and researchers diagnose and analyze the diseases and healthcare. Cancer diagnosis is very critical to potential patients since finding and treating cancers at an early stage can improve the survival rates. In this study, we use the EHRs obtained in clinics in New York City to classify the cancers. We train the support vector machine (SVM) model with medical records extracted from the EHRs for cancer classification. The model trained with 100 pieces of medical records per cancer, can achieve the prediction accuracy as $86.2 \%$ for 10 different types of cancers, and $97.33 \%$ for 3 types of cancers if trained with 400 pieces of medical records per cancer.
\end{abstract}

Keywords: electronic health records, cancer diagnosis, cancer classification, machine learning.

\section{INTRODUCTION}

An electronic health record (EHR) reports patients' clinic information, including patients' ages, genders, diagnoses, medications, allergies, treatment plans, immunization dates, radiology images, laboratory and lab test results, and medical history. EHRs are usually created and managed by authorized medical providers. Either the medical providers or the authorized personnel can access the EHR instantly and securely. EHRs can be shared to other organizations and health care providers involved in patients' care.

The medical and treatment histories of patients from EHRs are able to help medical providers diagnose and analyze patients' disease conveniently and efficiently. Because the information in EHRs is highly related to patients' health, it can be used for disease diagnosis and therapy. Jensen et al. (2012) used EHRs to improve research applications and clinical care. Kohane (2011) applied EHRs information in disease genomics studies. Persell et al. (2006) used EHRs to assess the validity of national quality measures for coronary artery.

Cancer is one of the deadliest diseases in the world. In 2017, 1,688,780 persons were diagnosed to have cancers and 600,920 cancer deaths were projected to happen in the United States (Siegel et al. 2017). Early diagnosis and treatment to cancer patients, are very important to improve the survival rates. Any delay or late therapy of cancers will reduce the survival rate and shorten the survival periods. EHRs can also provide 
valuable information in cancers diagnosis. Murphy et al. (2014) used EHRs to detect potential delays in cancer diagnosis. Singh et al. (2010) characterized and predicted the missed opportunities in lung cancer diagnosis trhough EHRs. Recently, the machine learning models have been widely applied in cancer diagnosis and prediction. Liao et al. (2017) used the random forest model on EHRs to classify different cancers. Kourou et al. (2015) applied machine learning techniques in cancer prognosis and prediction.

Although machine learning methods have been adopted in cancer diagnosis and prediction, there still exist some challenges. The quality of data set is one of the critical factors for supervised and unsupervised learning models. The models trained with wrong or biased data will result in low quality prediction. In order to have good classification and prediction results, machine learning models need sufficient training data. However, the medical data extracted from EHRs usually have a missing data problem, due to various reasons, including testing equipment availability, disease progression and others. The medical providers usually record patients' medical test results, medication, vital signs and information related to patients' health for several years, which is inevitable to have missing data problems in EHRs. As a result, the data extracted from EHRs with missing data problems, dramatically influence the performance of machine learning models in classification and prediction of diseases. Although, some studies about disease prediction have been done with machine learning models, the performance of machine learning models dealing with EHRs with missing data, has not been discussed. Therefore, it motivates us to study the performance of machine learning model on EHRs with missing data. Towards this goal, we use SVM technique on EHRs with missing data for cancer diagnosis and prediction, to evaluate the model performance and also show the application of EHRs effectively.

The rest of this paper is organized as followings. Section 2 introduces the related work on applications of EHRs and related techniques. Section 3 presents the SVM models used in this study. Section 4 describes the EHR data pre-processing. Section 5 presents the experimental result and discussion. Section 6 concludes the paper and points out the future work.

\section{RELATED WORK}

Recently, electronic health records have received much attention due to their potential advantages on medical diagnosis and analysis. Jensen et al. (2012) pointed out that clinical data describes the phenotypes and treatment of patients, and EHRs can be used to establish new patient-stratification principles for revealing unknown disease correlations. DesRoches et al. (2008) investigated the proportion of physicians who were using EHRs and the relationship between the adoption and the characteristics of individual physicians and their practices. The results indicated that the physicians who used EHRs believed such records improved the quality of care. Miriovsky et al. (2012) stated that rapidly accumulating clinical records can support cancer treatment and diagnosis. Therefore, EHR is a critical component of clinic evidence development and implementation.

Some researches and studies have been focusing on EHRs, due to the sufficient and valuable medical information related to patients' health extracting from EHRs. Zheng et al. (2017) proposed a semi-automated machine learning-based model to identify type-2 diabetes from the clinic data in EHRs. The proposed model was able to improve recall rate with a low false positive rate, while existing algorithms are usually suffering from a low recall rate and miss a lot of valuable samples under conservative filtering standards. Carroll et al. (2011) proposed to use the SVM model to identify Rheumatoid Arthritis cases by using medication and natural language processing-derived concepts on both expert-defined and naïve collections of EHR features. The SVM model was trained on naive and expert-defined data and the best performing naive system had precision of 0.94 and recall of 0.87 , compared with the precision of 0.75 and recall of 0.51 for the deterministic algorithm. This study indicated that the SVM model was capable to classify different disease cases from EHRs. Weiss et al. (2012) proposed to use machine learning techniques, such as SRL algorithm, relational functional gradient boosting, and outperforms propositional learners to predict primary myocardial infarction from EHRs. Garg et al. (2016) proposed to use machine learning models to identify rare diseases among a large number of other possible diagnoses. In their study, they identified a kind of rare 
diseases, cardiac amyloidosis, from EHRs by using an ensemble machine learning classifier. Liao et al. (2017) have used the random forest model on EHRs for cancers prediction. However, the accuracy is relatively low when the number of medical records for each cancer in the data set is small.

Although many methods were used in disease prediction, the studies on handling EHRs using machine learning were rare. One of the important issues is the missing data problem in EHRs. The missing data severely affects the implementation and efficiency when adopting machine learning methods. Comparing to the random forest model, SVM gains its popularity due to its interpretability and effectiveness. Therefore, in this paper, we propose to apply SVM models to EHRs with a large number of missing data for cancer classification and demonstrate its performance and effectiveness.

\section{BACKGROUND OF SUPPORT VECTOR MACHINE}

Support vector machine (SVM) is a supervised machine learning technique used for classification and regression analysis (Boser et al. 1992). Given a set of training points belonging to different classes, SVM could obtain a hyperplane to separate those points belonging to different classes.

The EHRs contain a large amount of medical records, which can be used as data points in SVM models. The SVM model can find a hyperplane to separate the medical records (data points) belonging to different cancers (classes). Figure 1 shows the example of a bi-classification problem. In the figure, the round and the star points are two kinds of data points (medical records) labeled with two different classes (cancers).

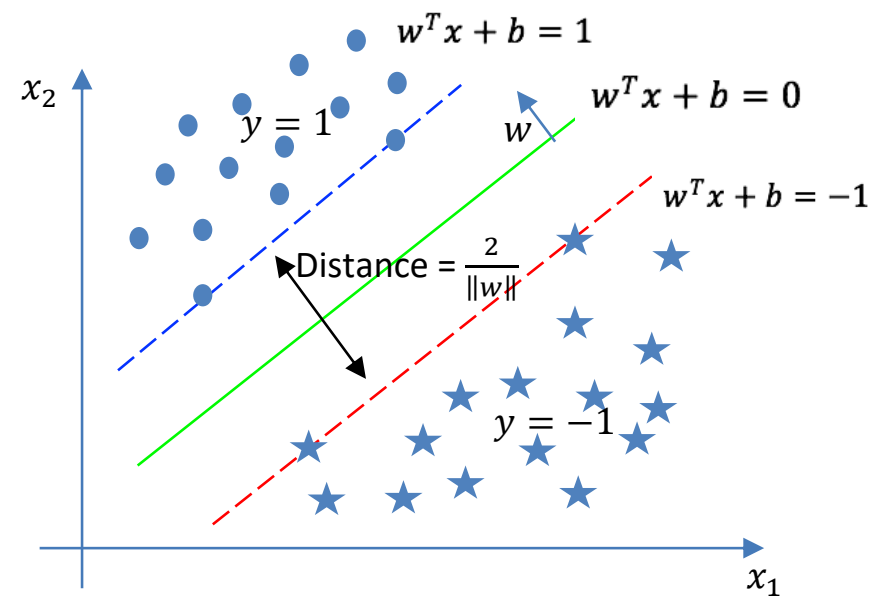

Figure 1: SVM for bi-classification.

If there are $n$ medical testing values in a piece of record, the data points should be a vector with dimension $n$. The label of each data point is the type of cancer. In Figure 1, $x, y$ represent the data points and corresponding labels respectively. The round and star points are labeled with 1 and -1 respectively. The SVM model is able to find a hyperplane, $\mathrm{w}^{\mathrm{T}} \mathrm{x}+\mathrm{b}=0$, where $x \in R^{n}$, to separate or classify those two types of data points $x$. The hyperplanes, $w^{T} x+b=0, w^{T} x+b=1$ and $w^{T} x+b=-1$ are on one or more data point. Those points are named support vectors, and that is the reason why the technique is called support vector machine. The normal of hyperplanes, $w$ in $\mathrm{w}^{\mathrm{T}} \mathrm{x}+\mathrm{b}=1$ and $\mathrm{w}^{\mathrm{T}} \mathrm{x}+\mathrm{b}=-1$, decides the perpendicular distance between those hyperplanes. SVM is able to find the suitable $w$ to make that distance largest by optimization. The distance between those two hyperplanes (shown as the blue and red dashed lined in Figure 1), can be calculated as $\frac{2}{\|w\|}$. So the problem turns to the optimal problems as Equation (1).

$$
\max \frac{1}{\|w\|}
$$




$$
\text { s.t. } y_{i}\left(w^{T} x_{i}+b\right) \geq 1, \quad i=1,2, \ldots n
$$

where: $y_{i}$ represent the label of $x_{i}, w$ is the normal vector of the hyperplane.

That optimization problem can be solved by minimizing $\|w\|^{2}$ by using lagrange multipliers method as shown in Equation (2):

$$
L(w, b, \lambda)=\frac{1}{2}\|w\|^{2}-\sum_{i=1}^{n} \lambda_{i}\left[y_{i}\left(w^{T} x_{i}+b\right)-1\right] \text { where } i=1,2, \ldots n,
$$

where: $\lambda_{i}$ is the lagrange multiplier.

The optimal value should be at the position $(w, b)$ with gradient equals to $\overrightarrow{0}$ (vector), which is described as Equation (3).

$$
\frac{\partial L}{\partial w}=0 \Rightarrow w=\sum_{i=1}^{n} \lambda_{i} y_{i} x_{i}, \quad \frac{\partial L}{\partial b}=0 \Rightarrow 0=\sum_{i=1}^{n} \lambda_{i} y_{i}
$$

After substituting the Equation (3) into (2), Equation (4) can be received as followings:

$$
\begin{aligned}
& L(w, b, \lambda)=\frac{1}{2}\|w\|^{2}-\sum_{i=1}^{n} \lambda_{i}\left[y_{i}\left(w^{T} x_{i}+b\right)-1\right] \\
& \quad=\frac{1}{2} w^{T} w-w^{T} \sum_{i=1}^{n} \lambda_{i} y_{i} x_{i}-b \sum_{i=1}^{n} \lambda_{i} y_{i}+\sum_{i=1}^{n} \lambda_{i} \\
& \quad=-\frac{1}{2}\left(\sum_{i=1}^{n} \lambda_{i} y_{i} x_{i}\right)^{T} \sum_{i=1}^{n} \lambda_{i} y_{i} x_{i}+\sum_{i=1}^{n} \lambda_{i} \\
& =-\frac{1}{2} \sum_{i, j=1}^{n} \lambda_{i} \lambda_{j} y_{i} y_{j} x_{i}^{T} x_{i}+\sum_{i=1}^{n} \lambda_{i} .
\end{aligned}
$$

The optimal $w$ and $b$ can be obained after solving the upper problem. Finally, the optimal normal $w$ and $b$ define a hyperplane, which guarantees the distance between the hyperplanes is largest, shown as the blue and red dashed line in Figure 1.

\section{DATA PRE-PROCESSING}

The EHRs used in this study, are obtained from clinics in New York City. The EHRs contain different components, including laboratory testing results, patient's vital signs, patient IDs, genders and ages, and the disease names described in ICD-9 or ICD-10 code. We initially create vectors to store the clinic data for each patient in EHRs. The entries of each vector store the laboratory testing results, patients' information, vital signs and other data from one clinic visit. The patient IDs, laboratory testing dates, service dates and other unrelated information will be deleted from the vectors. We only keep the ICD-9 or ICD-10 code, the laboratory testing result and patients' vital signs. After that, each disease is corresponded to multiple vectors storing medical data. All the vectors collected from the EHRs were gathered together as the dataset for machine learning models. Each row and column in dataset are corresponded to a data point and a feature in model training and prediction respectively. However, those datasets are usually imbalanced due to the distribution of different cancers. In the EHRs we used in this study, there are totally 24 cancers with 28 features. The "Cancer (Carcinoma) of breast, female" has 2,267 samples labeled as 13 in Figure 2, and the "Brain tumor" only has 1 sample labeled as 23 . Figure 2 shows the distribution of diseases. In the figure, the horizontal axis is the index of different types of cancers and the vertical axis is the number of medical records. From the figure we see that the numbers of various cancers are very unbalanced. Table 1 shows the number of records of each cancer. The number of different cancer is highly unbalanced. In order to 
balance the data points, we set a threshold to filter the cancers. Only the cancer with enough records will be used, and randomly take the same number of records for each cancer as the dataset.

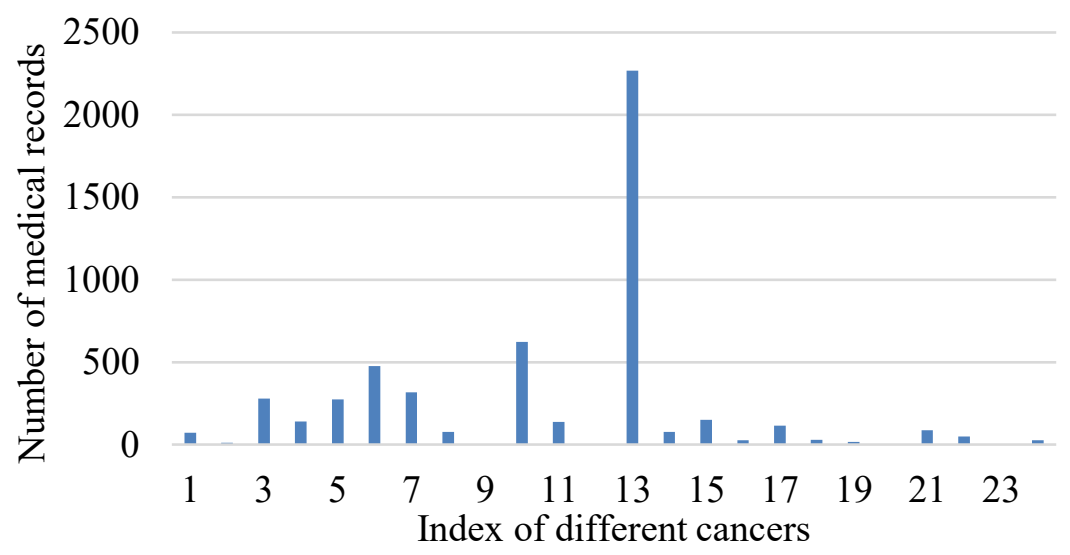

Figure 2: The distribution of number of medical records for different types of cancers.

Table 1: Number of records for different cancers.

\begin{tabular}{|c|c|c|c|c|c|}
\hline $\begin{array}{l}\text { ICD } \\
\text { code }\end{array}$ & Cancer Name & Number & $\begin{array}{l}\text { ICD } \\
\text { code }\end{array}$ & Cancer Name & Number \\
\hline 142.9 & $\begin{array}{l}\text { Malignant neoplasm of sali- } \\
\text { vary gland }\end{array}$ & 72 & 145.9 & $\begin{array}{l}\text { Cancer of mouth, Malignant } \\
\text { neoplasm of mouth }\end{array}$ & 11 \\
\hline 174.9 & Cancer of breast & 2267 & 180.9 & Cancer (Carcinoma) of cervix & 78 \\
\hline 147.9 & $\begin{array}{l}\text { Malignant neoplasm of naso- } \\
\text { pharynx }\end{array}$ & 278 & 161.9 & $\begin{array}{c}\text { Malignant neoplasm of lar- } \\
\text { ynx }\end{array}$ & 2 \\
\hline 150.9 & Cancer of esophagus & 139 & 188.9 & Bladder cancer & 25 \\
\hline 151.9 & Cancer of stomach & 273 & 189.1 & Renal pelvis cancer & 115 \\
\hline 153.9 & Cancer of colon & 475 & 191.9 & Malignant neoplasm of brain & 30 \\
\hline 154.1 & Cancer of rectum & 318 & 193 & Cancer of thyroid gland & 16 \\
\hline 157.9 & Cancer of pancreas & 76 & 198.3 & Metastatic brain tumor & 1 \\
\hline 185 & Cancer of prostate & 149 & 198.5 & Bone metastasis & 88 \\
\hline 162.9 & Cancer of lung & 624 & 202.8 & Lymphoma & 49 \\
\hline 171.9 & $\begin{array}{c}\text { Cancer of connective and } \\
\text { soft tissue }\end{array}$ & 137 & 172.9 & $\begin{array}{l}\text { Malignant melanoma, Malig- } \\
\text { nant melanoma of skin }\end{array}$ & 1 \\
\hline 239.6 & Brain tumor & 1 & 239.7 & Spinal cord tumor & 26 \\
\hline
\end{tabular}

However, the dataset contains the missing value, which will influence the quality of model training and lower the prediction accuracy. EHRs usually have such problems since it cannot guarantee different patients have all the laboratory tests and vital sign measurements. In order to figure out that problem, we use mean values to replace the missing values for each feature (a laboratory testing or a vital sign) with missing data in each cancer. The following experimental results show the feasibility of this strategy. 


\section{EXPERIMENTS AND RESULTS}

In this study, We use SVM models with linear and Radial Basis Function (RBF) kernels to classify and predict different cancers by using the medical data extracted from EHRs. Our goal is to estimate how effectively the SVM model could work on EHRs. We also use $K$ - fold cross-validation technique to estimate the performance of model with different parameters. Cross-validation is a resampling method used to evaluate machine learning models. The $K$ - fold validation divide the data in to $K$ portions, and train and test the model by $K$ rounds. Each time, the model is trained by $K-1$ portion of data and tested with the rest of data (one portion). That portion of data for testing is different during $K$ times testing. For example, the data except the first portion, is used for training and the first portion is used for testing in the first round of training and testing. In the second round, the second portion of data is used for testing, and the rest portions are used for training. All the data will be evenly used for testing and training after $K$ rounds. $K$ - fold validation will obtain $K$ testing scores after $K$ rounds of running. Because, one testing score will be obtained after one round of model training and testing. The average of $K$ testing scores will be the final testing score. The $K$ - fold cross validation is able to prevent the over fitting problem since it is possible that the model could occasionally get a high training score and low testing score because the selected training data contains less information about the testing data. As a result, the trained model cannot differentiate the testing data, even it gets high training score, which is over fitting. By using the $K$-fold cross validation, the data can be evenly used in training and testing, and the resultant average testing score is upon all data, which could guide the tunings on model parameters to prevent over fitting problems. We use 10- fold cross validation during the model training and testing in this study.

We use the linear and RBF kernel SVM models trained with different number of training sample and then compare the testing scores to evaluate the influence of number of training sample on prediction accuracy. Figure 3 shows the testing scores of linear and RBF kernel of SVM models trained with different number of data points. In the figure, the horizontal axis represents the number of data points, which is used to filter the number of types of cancers. If the number of data entries for a cancer is below a certain number (100, 200,300 , or 400), we will not use its data. The vertical axis is the testing score, representing the prediction accuracy. The red bars mean the testing scores for the linear SVM model, and the blue bars are the testing scores of the RBF kernel SVM model. From the figure, we know that the RBF kernel SVM model is more effective since it achieves higher testing scores for all the cases. With the increase of the number of data points, less number of cancers and their data are selected. However, the data entries for each cancer is large, and therefore testing scores are higher. 


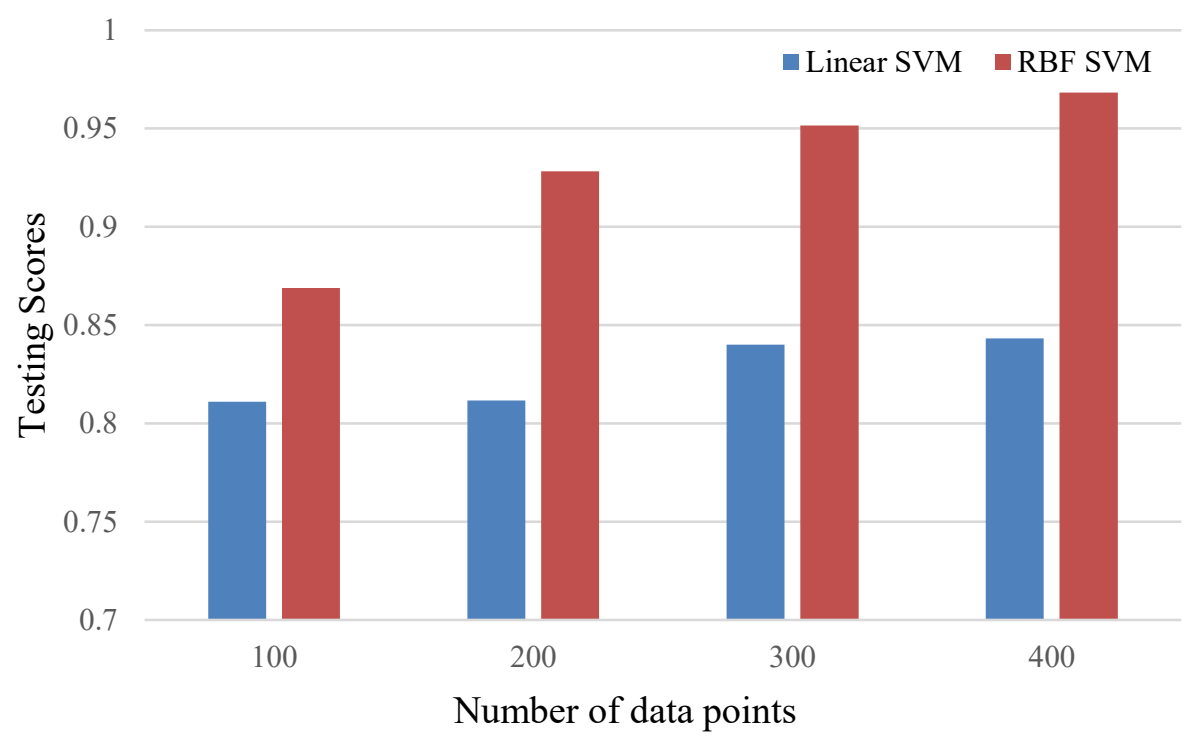

Figure 3: The testing scores of linear and RBF kernel SVM models trained with different number of data points (10-fold cross validation).

Figure 4 shows the testing scores of the linear and RBF kernel SVM models trained with the 300 data points for each type of cancer and different $K$-fold cross validation $(K=2 \sim 30)$. In the figure, the horizontal axis and the vertical axis are the value of $K$ used in the validation and the testing scores. We use the red bars and blue bars to represent the test scores of the linear SVM model and the RBK SVM model respectively. From the figure, we can see that the RBF kernel SVM model is more efficient than the linear SVM model. A bigger value of $K$ can make the testing scores stable. In this case, the testing scores are stable if $K$ is larger than 20. This is because the training and testing data are used more evenly if the value of $K$ is larger.

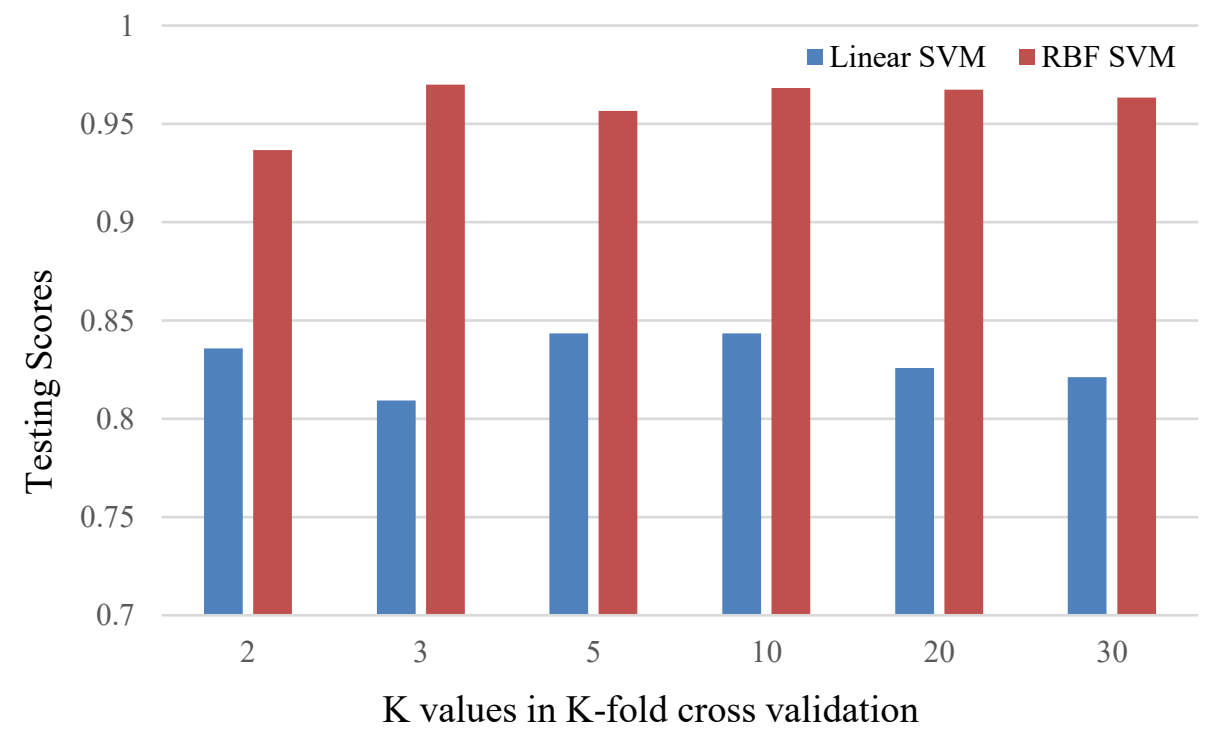

Figure 4: The testing scores of linear and RBF kernel SVM models trained with different $K$ of $K$ - fold cross validation.

Figure 5 shows the top ten feature importance used in the SVM model with linear kernel. In the figure, the horizontal axis represents the type of features, and the vertical axis represents the importance of weight. 
From the figure, we know that glucose, glomerular, and globulin are the top three factors for cancer prediction. The features with high importance are those key factors in cancer prediction and classification. Hence, the importance of features could provide critical information on cancer diagnosis and prediction. It is impossible to obtain the feature importance from the model with RBF kernel because the RBF kernel converts the original feature space to kernel space, and the importance of weights in kernel space is not relevant to the original feature space.

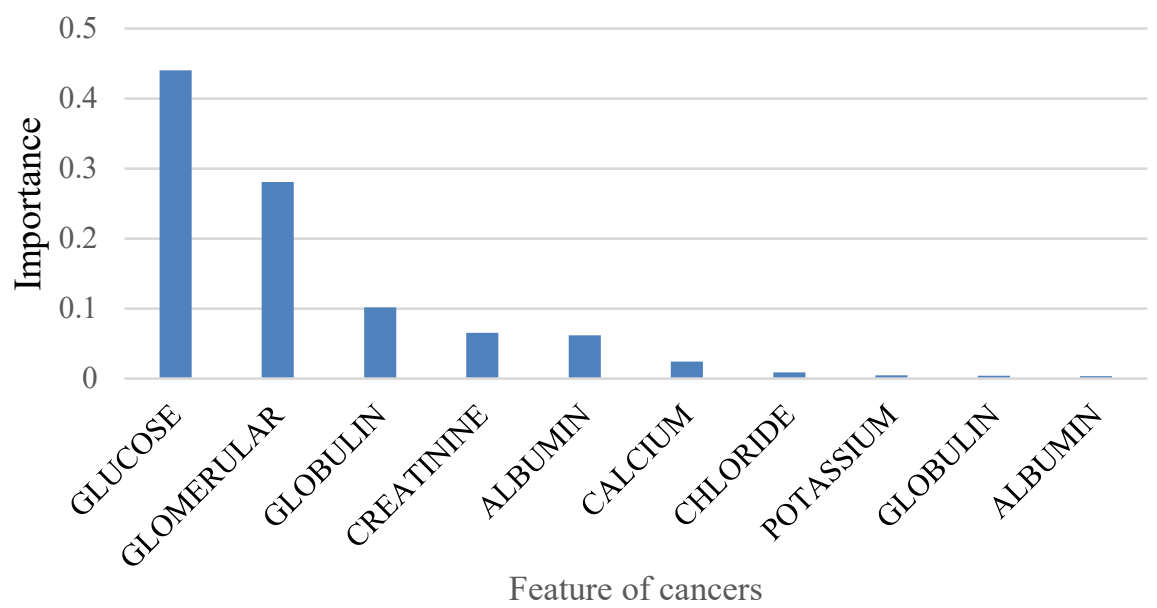

Figure 5: The top ten feature importance for classification cancers.

\section{CONCLUSIONS AND FUTURE WORK}

In this study, we used SVM models to classify cancers based on the medical data extracted from EHRs. The prediction accuracy is $97.33 \%$ when classifying 3 types of cancers with 400 medical records for each cancer, and $86.2 \%$ when classifying 10 types of cancers with 100 medical records for each cancer. We also calculated the importance of each feature used in SVM model with linear kernel for cancer prediction. The features (or medical tests) with higher importance are critical in predicting the potential of cancer disease. The model may achieve higher prediction accuracy if a larger number of medical records are used. We will continue this work along the following directions. First, we will develop more advanced methods to handle missing data for cancer classification. Second, the systematic analysis and comparison will be conducted for various machine learning approaches for EHRs. Third, we plan to develop a framework to process EHRs and apply machine learning and deep learning methods for disease prediction and apply them to larger data sets for further validation.

\section{REFERENCES}

Boser, B. E., I. M. Guyon and V. N. Vapnik. 1992. "A training algorithm for optimal margin classifiers". In Proceedings of the fifth annual workshop on Computational learning theory pp 144-152.

Carroll, R. J., A. E. Eyler and J. C. Denny. 2011. "Naïve electronic health record phenotype identification for rheumatoid arthritis". In AMIA annual symposium proceedings pp 189.

DesRoches, C. M., E. G. Campbell, S. R. Rao, K. Donelan, T. G. Ferris, A. Jha, R. Kaushal, D. E. Levy, S. Rosenbaum and A. E. Shields. 2008. "Electronic health records in ambulatory care-a national survey of physicians". New England Journal of Medicine Vol. 359(1), pp. 50-60. 
Garg, R., S. Dong, S. Shah and S. R. Jonnalagadda. 2016. "A bootstrap machine learning approach to identify rare disease patients from electronic health records". arXiv preprint arXiv:1609.01586 Vol. pp.

Jensen, P. B., L. J. Jensen and S. Brunak. 2012. "Mining electronic health records: towards better research applications and clinical care". Nature Reviews Genetics Vol. 13(6), pp. 395.

Kohane, I. S. 2011. "Using electronic health records to drive discovery in disease genomics". Nature Reviews Genetics Vol. 12(6), pp. 417.

Kourou, K., T. P. Exarchos, K. P. Exarchos, M. V. Karamouzis and D. I. Fotiadis. 2015. "Machine learning applications in cancer prognosis and prediction". Computational and structural biotechnology journal Vol. 13(pp. 8-17.

Liao, S., J. Xiao, Y. Xie and F. Gu. 2017. "Towards use of electronic health records: cancer classification". In Proceedings of the Symposium on Modeling and Simulation in Medicine pp 4.

Miriovsky, B. J., L. N. Shulman and A. P. Abernethy. 2012. "Importance of health information technology, electronic health records, and continuously aggregating data to comparative effectiveness research and learning health care". Journal of Clinical Oncology Vol. 30(34), pp. 4243-4248.

Murphy, D. R., A. Laxmisan, B. A. Reis, E. J. Thomas, A. Esquivel, S. N. Forjuoh, R. Parikh, M. M. Khan and H. Singh. 2014. "Electronic health record-based triggers to detect potential delays in cancer diagnosis". BMJ Qual Saf Vol. 23(1), pp. 8-16.

Persell, S. D., J. M. Wright, J. A. Thompson, K. S. Kmetik and D. W. Baker. 2006. "Assessing the validity of national quality measures for coronary artery disease using an electronic health record". Archives of Internal Medicine Vol. 166(20), pp. 2272-2277.

Siegel, R. L., K. D. Miller and A. Jemal. 2017. "Cancer statistics, 2017". CA: a cancer journal for clinicians Vol. 67(1), pp. 7-30.

Singh, H., K. Hirani, H. Kadiyala, O. Rudomiotov, T. Davis, M. M. Khan and T. L. Wahls. 2010. "Characteristics and predictors of missed opportunities in lung cancer diagnosis: an electronic health record-based study". Journal of Clinical Oncology Vol. 28(20), pp. 3307.

Weiss, J. C., S. Natarajan, P. L. Peissig, C. A. McCarty and D. Page. 2012. "Machine learning for personalized medicine: Predicting primary myocardial infarction from electronic health records". AI Magazine Vol. 33(4), pp. 33.

Zheng, T., W. Xie, L. Xu, X. He, Y. Zhang, M. You, G. Yang and Y. Chen. 2017. "A machine learningbased framework to identify type 2 diabetes through electronic health records". International journal of medical informatics Vol. 97 pp. 120-127.

\section{AUTHOR BIOGRAPHIES}

XUDONG ZHANG is a PhD student in the Department of Computer Science at the Graduate Center of The City University of New York. His research interests include machine learning, bioinformatics, modeling and simulation, and high performance computing. His email address is xzhang5@gradcenter.cuny.edu.

JIEHAO XIAO is a PhD student in the Department of Computer Science at the Graduate Center of The City University of New York. His research interests include machine learning and medical simulation. His email address isjxiao@gradcenter.cuny.edu.

FENG GU is an Assistant Professor in the Department of Computer Science at College of Staten Island, The City University of New York. He holds a Ph.D. in Computer Science from Georgia State University. His research interests include modeling and simulation, high performance computing, and bioinformatics. His email address is Feng.Gu@csi.cuny.edu. 